\title{
Revascularização transmiocárdica a laser
}

\author{
Maurício GALANTIER*, Geisha Barbosa MOREIRA*, Rolf Francisco BUB*, João GALANTIER*, \\ Ênio BUFFOLO*, Antônio Carlos CARVALHO*, Dikran ARMAGANIJAN**, Jozef FÉHER*
}

Galantier M, Moreira G B, Bub R F, Galantier J, Buffolo E, Carvalho A C, Armaganijan D, Féher J Revascularizaçāo transmiocárdica a laser. Rev Bras Cir Cardiovasc 1996; 11 (2): 67-74.

RESUMO: A doença coronária aterosclerótica apresenta situaçōes em que as técnicas cirúrgicas habituais ou as realizadas em laboratórios de hemodinâmica não são passiveis de utilização. São pacientes portadores de comprometimento grave e difuso das artérias coronárias, mas ainda com preservação da viabilidade miocárdica e que evoluem com importante sintomatologia anginosa, apesar da terapêutica clínica adequada. Técnicas de endarterectomia são preconizadas em algumas situaçōes, porém com resultados trans e pós-operatórios muitas vezes desfavoráveis. Em outras ocasiōes pode, inclusive, ser necessária a indicação de transplante cardiaco. Para este grupo de pacientes uma nova abordagem vem sendo desenvolvida a partir dos estudos do Dr. Mirhoseini, com a confecção de vários túneis transmiocárdicos com o uso de raios laser de $\mathrm{CO}_{2}$ de alta potência (850 watts). Os autores relatam sua experiência clínica inicial no Hospital Israelita Albert Einstein, em São Paulo, com a utilização da Revascularização Transmiocárdica a laser (Heart Laser) no período de abril de 1995 a fevereiro de 1996. Foram operados 11 pacientes com essa técnica (em um deles foi utilizada revascularizaçăo associada), sendo a média de idades de 68 anos. Sete deles eram casos de reoperaçōes. Desses pacientes, 9 encontravam-se em grupo funcional IV. Houve 2 óbitos no periodo pós-operatório, ambos em pacientes com idade superior a 80 anos. Sete pacientes foram avaliados com 3 meses de pós-operatório, mostrando melhora clínica e maior tolerância a esforços. Em 4 pacientes foi realizado mapeamento cardiaco com isótopos e, em 1, houve melhora da perfusão. Os resultados, embora promissores, ainda requerem uma avaliaçăo mais prolongada em um maior grupo de pacientes, para que sua utilização seja melhor estabêlecida.

DESCRITORES: Revascularização do miocárdio, métodos. Cirurgia a laser, métodos. Isquemia miocárdica, cirurgia. Angina pectoris, cirurgia. Arteriosclerose coronária, cirurgia. Aterosclerose coronária, cirurgia.

\section{INTRODUÇÃO}

O tratamento da insuficiência coronária tem sido realizado com indicações e resultados já estabelecidos, tanto através de cirurgia, com pontes venosas, ou enxertos arteriais utilizando a artéria torácica interna, artéria radial, artéria epigástrica inferior, artéria gastro-epiplóica ${ }^{(1-8)}$ ou, ainda, pelos vários procedimentos hemodinâmicos, como a angioplastia transluminal coronária e métodos mais recentes, como a aterectomia direcionada ou rotacional, o laser e os stents ${ }^{(3,9-12)}$. Em situações nas quais o acometimento aterosclerótico das coronárias é mais difuso, com estenoses distais e significativas, não permitindo o tratamento tradicional, faz-se necessária a realização de procedimentos como a endarte-

Trabalho realizado no Hospital Israelita Albert Einstein. São Paulo, SP, Brasil.

Apresentado ao $23^{\circ}$ Congresso Nacional de Cirurgia Cardiaca. Recife, PE, 21 a 23 de março, 1996.

- Do Hospital Israelita Albert Einstein.

* Do Instituto Dante Pazzanese de Cardiologia, São Paulo, SP.

Endereço para correspondência: Maurício Galantier. Rua Constantino de Souza, 1025, 14ª andar. CEP: 04605-003. São Paulo, SP, Brasil. Tel. [011] 543.1640 e 842.3265 
rectomia coronária, cujos resultados são ainda discutíveis $(13,14)$ ou, mesmo, o transplante cardíaco.

Métodos de revascularização indireta do miocárdio, objetivando melhorar a perfusão do músculo cardíaco através da via extracoronariana, já foram utilizados há muitos anos, antes mesmo do advento da cinecoronariografia, como a escarificação epicárdica, a talcagem pericárdica, a omentopexia (15) e a acupuntura miocárdica (16). O implante de artéria torácica interna, proposta por VINEBERG (17) teve seus resultados comprovados pela injeção de contraste nas artérias implantadas, mostrando, em vários pacientes, a sua conexão através dos sinusóides miocárdicos (descritos por WEARN et al. (18) com porções distais das coronárias (19). Essa técnica foi relegada a plano secundário na evolução da cirurgia coronária, uma vez que levava meses até que a circulação se desenvolvesse e também porque inúmeros pacientes voltavam a apresentar sintomatologia. Baseados nestas antigas experiências de neovascularização do miocárdio isquêmico, e também na análise de répteis que apresentam circulação coronária muito pobre mas evidenciam túneis intramiocárdicos originários a partir da cavidade ventricular MIRHOSEINI et al. (20) e, posteriormente, outros pesquisadores como COOLEY et al. (21) e HORVATH et al. (22) desenvolveram modelos experimentais em animais nos quais ligavam ramos coronários e, a seguir, aplicavam feixes de raios laser de $\mathrm{CO} 2$ de alta potência (850 watts) a partir do epicárdio em direção à cavidade ventricular, criando canais transmiocárdicos. A avaliação desses animais comparados a outros, em que apenas se ligavam ramos coronários ou ligaduras temporárias, mostrou que as áreas de infarto eram significativamente menores nos grupos tratados por laser e em que os canais permaneciam patentes por longo período.

Esses fatos fizeram com que a utilização clínica da revascularização transmiocárdica com uso de raios laser fosse protocolada, sendo que, atualmente, 25 Centros em todo o mundo têm realizado tal técnica. Em abril de 1995, iniciamos sua utilização em um grupo de pacientes cujos resultados preliminares apresentamos neste trabalho.

\section{CASUÍSTICA E MÉTODOS}

Entre abril de 1995 e fevereiro de 1996, foram tratados com a técnica de revascularização transmiocárdica a laser, no Hospital Israelita Albert Einstein, 11 pacientes.

Os dados para a inclusão neste estudo foram:

1) Pacientes com importantes sintomas de angina (grupo funcional III ou IV), apesar de tratamento médico adequado.
2) Anatomia coronária não favorável à revascularização tradicional ou, mesmo, a procedimentos hemodinâmicos.

3) Pacientes com viabilidade miocárdica nas áreas isquêmicas, comprovada por técnicas como estudos utilizando SESTAMIBI TC $99 \mathrm{M}$ ou por ecocardiograma farmacológico com o uso de dobutamina.

4) Pacientes em condiçṑes clínicas favoráveis à realização de toracotomia, ou aqueles não portadores de doenças limitantes da sobrevida (idade avançada, doenças malignas em atividade etc).

Desses pacientes, 9 eram do sexo masculino, com idade variando de 49 a 87 anos, com média de 68 anos. Sete $(63 \%)$ haviam sido submetidos a pelo menos uma operação de revascularização do miocárdio e $3(27 \%)$ a uma segunda. Angioplastia coronária havia sido realizada em 5 pacientes. (Tabela 1).

TABELA 1

\begin{tabular}{lc}
\hline SEXO & MASCULINO 9 \\
\hline Idade & 68 anos (49 a 87) \\
Cirurgia Prévia & 7 \\
Angioplastia Prévia & 5 \\
\hline
\end{tabular}

Todos os pacientes apresentavam importante sintomatologia anginosa. Nove estavam em grupo funcional IV e 2 em grupo funcional III. Três pacientes apresentavam algum grau de insuficiência cardíaca e outros 3 apresentavam quadro anginoso instável, necessitando uso de medicação endovenosa. Todos recebiam nitratos, inibidores dos canais de cálcio, betabloqueadores (exceto 1, portador de bronquite asmática) e antiadesivos plaquetários.

Os pacientes apresentavam lesões importantes e difusas nos três territórios coronarianos, não sendo considerados como candidatos a revascularização tradicional ou procedimento hemodinâmico terapêutico.

Todos os pacientes foram submetidos a teste ergométrico com uso de SESTAMIBI TC $99 \mathrm{M}$, sendo que 3 não conseguiram realizar nenhum tipo de exercício, e 7 interromperam o teste no final do $1^{\circ}$ ou início do $2^{\circ}$ estágio (protocolo de Bruce modificado).

Também foram submetidos a ecocardiografia pré-operatória, sendo que a fração de ejeção variou entre 0,31 e 0,75 (com média de 0,50 ). Os últimos 8 pacientes foram submetidos a estudo farmacológico com uso de dobutamina, para melhor avaliação da viabilidade miocárdica.

Com a interpretação conjunta da cinecorónariografia, cintilografia miocárdica e ecocardiograma 
Galantier M, Moreira G B, Bub RF, Galantier J, Buffolo E, Carvalho A C, Armaganijan D, Féher J - Revascularização transmiocárdica a laser. Rev Bras Cir Cardiovasc 1996; 11 (2): 67-74.

TABELA 2

AREAS MAIS COMPROMETIDAS

\begin{tabular}{ll} 
Todas & 3 \\
Anterior e Lateral & 5 \\
Lateral e Inferior & 3 \\
\hline
\end{tabular}

farmacológico, efetuamos a análise das áreas isquêmicas mais comprometidas. (Tabela 2).

Após monitorização contínua da pressão arterial, eletrocardiograma e uso de cateter de Swan Ganz, os pacientes foram anestesiados e intubados com cânula endotraqueal de duplo lúmen. Foi realizado estudo com ecocardiograma transesofágico, para análise da função mocárdica global e grau de regurgitação mitral. Todos os pacientes, exceto 1 , foram operados através de toracotomia ântero-lateral esquerda, no $4^{\circ}$ ou $5^{\circ}$ espaço intercostal. O pericárdio foi aberto anteriormente ao nervo frênico. Nos casos de cirurgia prévia, as aderências foram desfeitas, cuidando-se em preservar eventuais enxertos funcionantes.

O equipamento de laser (The Heart Laser TM $\mathrm{CO}_{2}$ Surgical Laser System)* era ajustado para disparos entre 36 e 45 joules, com largura de pulso de 45 a 50 milisegundos e sincronizados ao ECG de forma que ocorressem no pico da onda R, quan-

- Laser Engineering Inc do o ventrículo esquerdo estivesse totalmente distendido e em período refratário absoluto.

A energia do laser é absorvida pelo sangue no interior do ventrículo e isso produz uma imagem acústica semelhante a bolhas, que pode ser captada pelo ecocardiograma transesofágico. Esse fato, assim como a evidência do sangramento pela perfuração do laser foram os critérios adotados para avaliar a eficácia do disparo. Foram realizados em média 30 disparos eficazes, sendo que as áreas com fibrose ou maior depósito de gordura eram as que ofereciam maiores dificuldades à perfuração adequada.

A hemostasia das perfurações era obtida pela compressão digital e raramente houve necessidade de sutura. O sangue aspirado era em geral processado em equipamento "Cell Saver" para ser reinfundido.

Após hemostasia adequada, o tórax foi drenado e a toracotomia reconstruida com a técnica habitual.

Em 1 paciente, a toracotomia foi mediana transesternal e, neste, além da revascularização a laser (território da coronária esquerda), foi realizada anastomose da torácica interna com a coronária direita (enxerto livre) sem o uso de circulação extracorpórea.

Os pacientes foram avaliados clinicamente no período de pós-operatório e também por estudo com teste ergométrico associado a cintilografia miocárdica e ecocardiografia farmacológica.

GRÁFICO 1

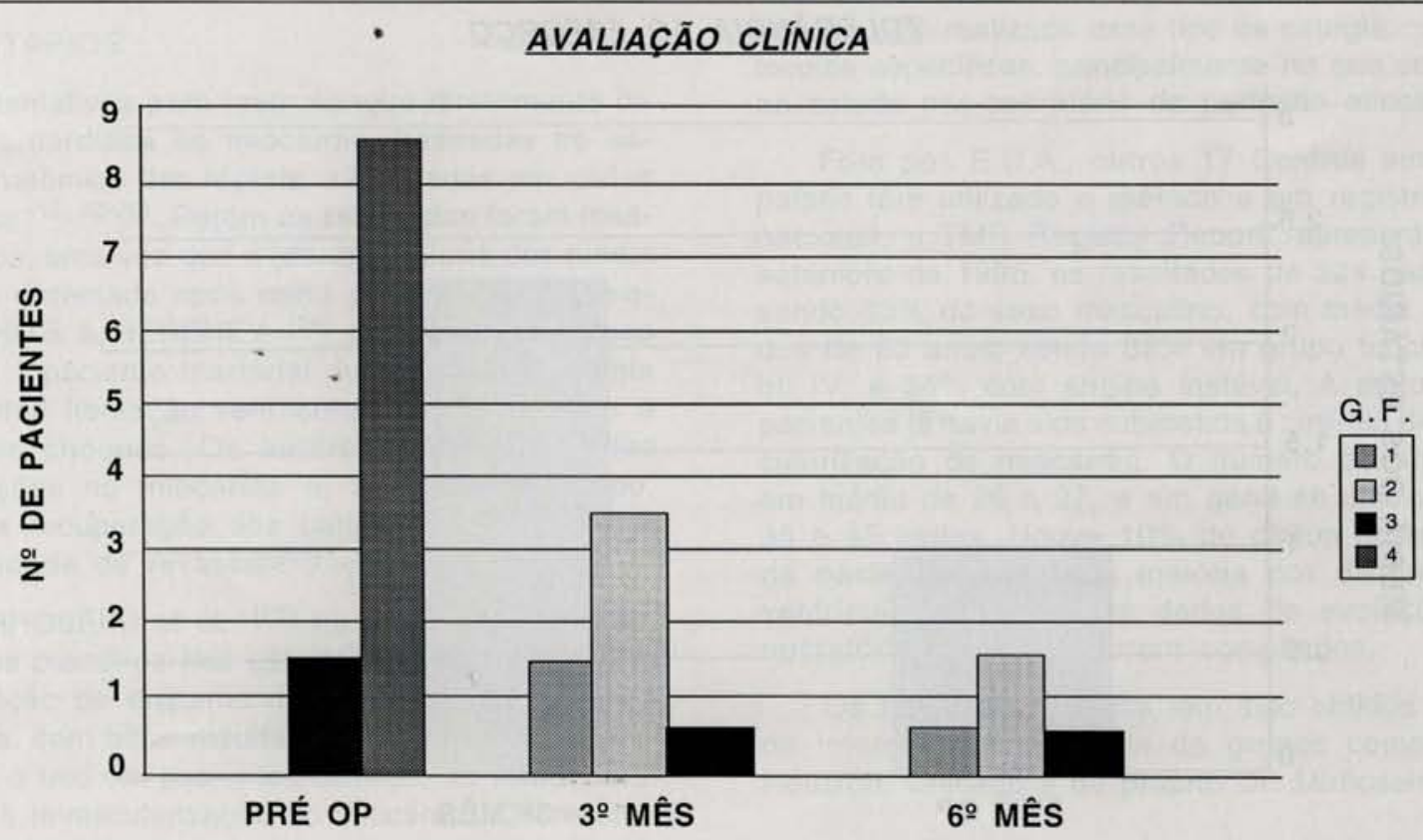




\section{RESULTADOS}

A morbidade do procedimento foi baixa e pouco grave: 1 paciente apresentou secreção e deiscência de sutura, necessitando curativos e ressutura; 1 paciente apresentou enfisema subcutâneo de resolução expontânea e 1 paciente apresentou estase veno-capilar no $3^{\circ}$ dia de P. O., necessitando uso de diuréticos e drogas hipotensoras. Não houve casos de reoperação por sangramento. A permanência na UTI foi de 1 a 2 dias, com mais de $60 \%$ dos pacientes recebendo alta da UTI no dia seguinte à cirurgia. Em todos os pacientes a medicação pré-operatória foi mantida.

Houve 2 óbitos hospitalares. Um paciente, com 87 anos, apresentou instabilidade hemodinâmica nas primeiras horas de pós-operatório, necessitando drogas inotrópicas. Após ser estabilizado, não conseguiu se manter sem suporte respiratório e, após três dias, mostrou sinais de infecção, com evolução para quadro séptico e óbito no quinto dia de pósoperatório. Outro paciente, com 82 anos, apresentou na quinta hora de P. O. (anteriormente estava estável, inclusive com tendência a hipertensão) hipotensão arterial rebelde às drogas vasoativas; não havia sinais de tamponamento cardiaco ou presença de hemorragia significativa; após estabilização, desenvolveu quadro neurológico com hemiplegia esquerda e suspeita de infarto lateral. Voltou a apresentar instabilidade hemodinâmica, com óbito no quarto dia de pós-operatório.

Sete pacientes foram avaliados clinicamente com 3 meses, e 4 com 6 meses de pós-operatório. Houve melhora clínica em todos, passando de grupo funcional médio de 3,8 para 1,8 com 3 meses e 1,7 com 6 meses (Gráfico 1).

Quatro pacientes efetuaram cintilografia miocárdica de esforço com três meses de pós-operatório. A tolerância ao esforço melhorou, tendo os pacientes conseguido efetuar dois a três estágios, sendo que 1 deles completou o quarto estágio do teste de Bruce (Gráfico 2).

Em relação à captação de isótopos, em 1 paciente houve melhora em parede lateral; em outro não foi possível comparação, uma vez que o mesmo não conseguiu realizar esforço pré-operatório. Outro paciente não mostrou melhora na captação na parede livre do ventrículo esquerdo, porém apresentou evidente hipocaptação no septo distal (região não tratada pelo laser) e outro não apresentou alterações na captação em relação ao pré-operatório (Gráfico 3).

Dado significativo foi a melhora imediata obtida em alguns pacientes. Dois deles, que se encontravam muito sintomáticos, com uso diário de 2 a 3

GRÁFICO 2

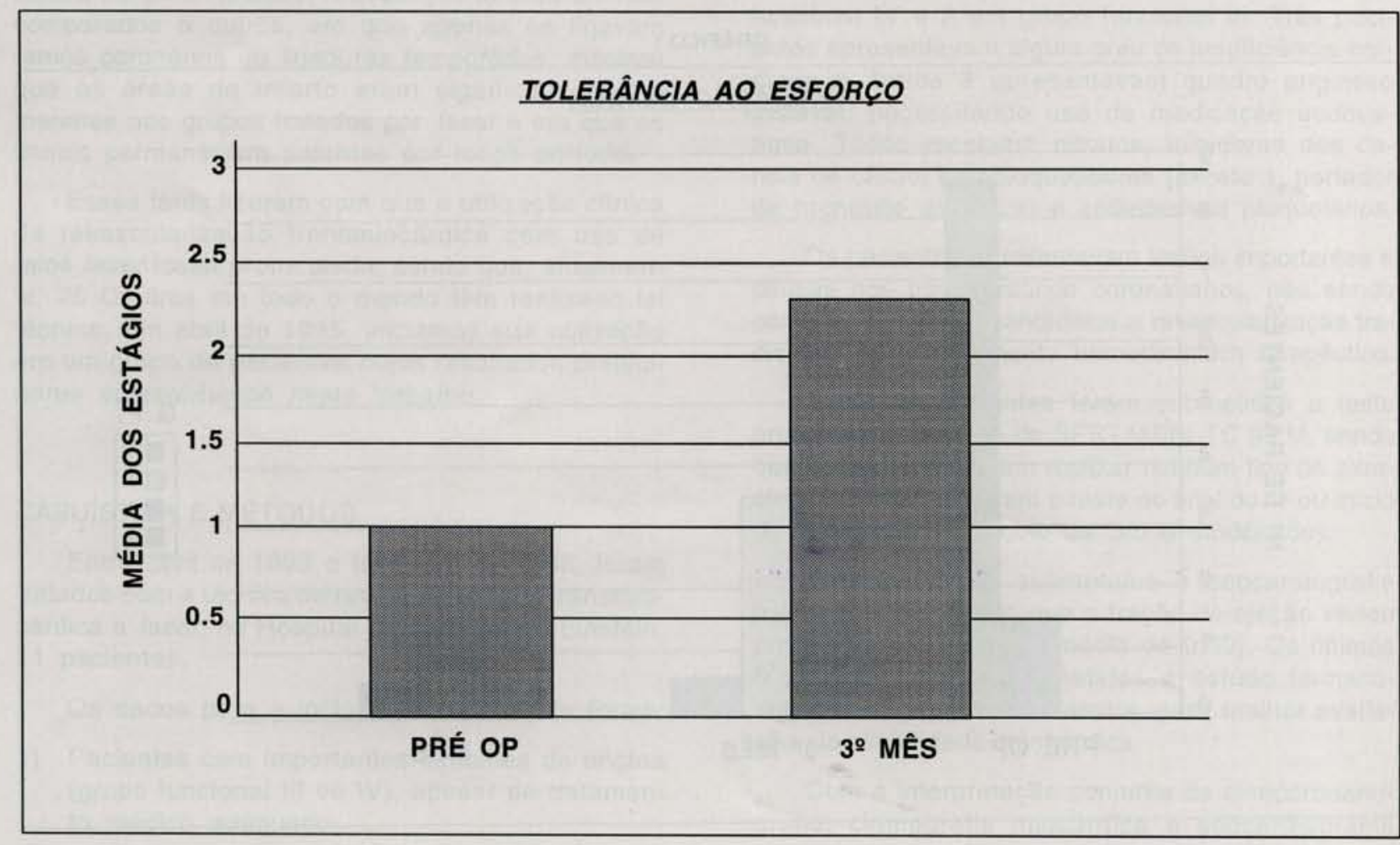


GRÁFICO 3

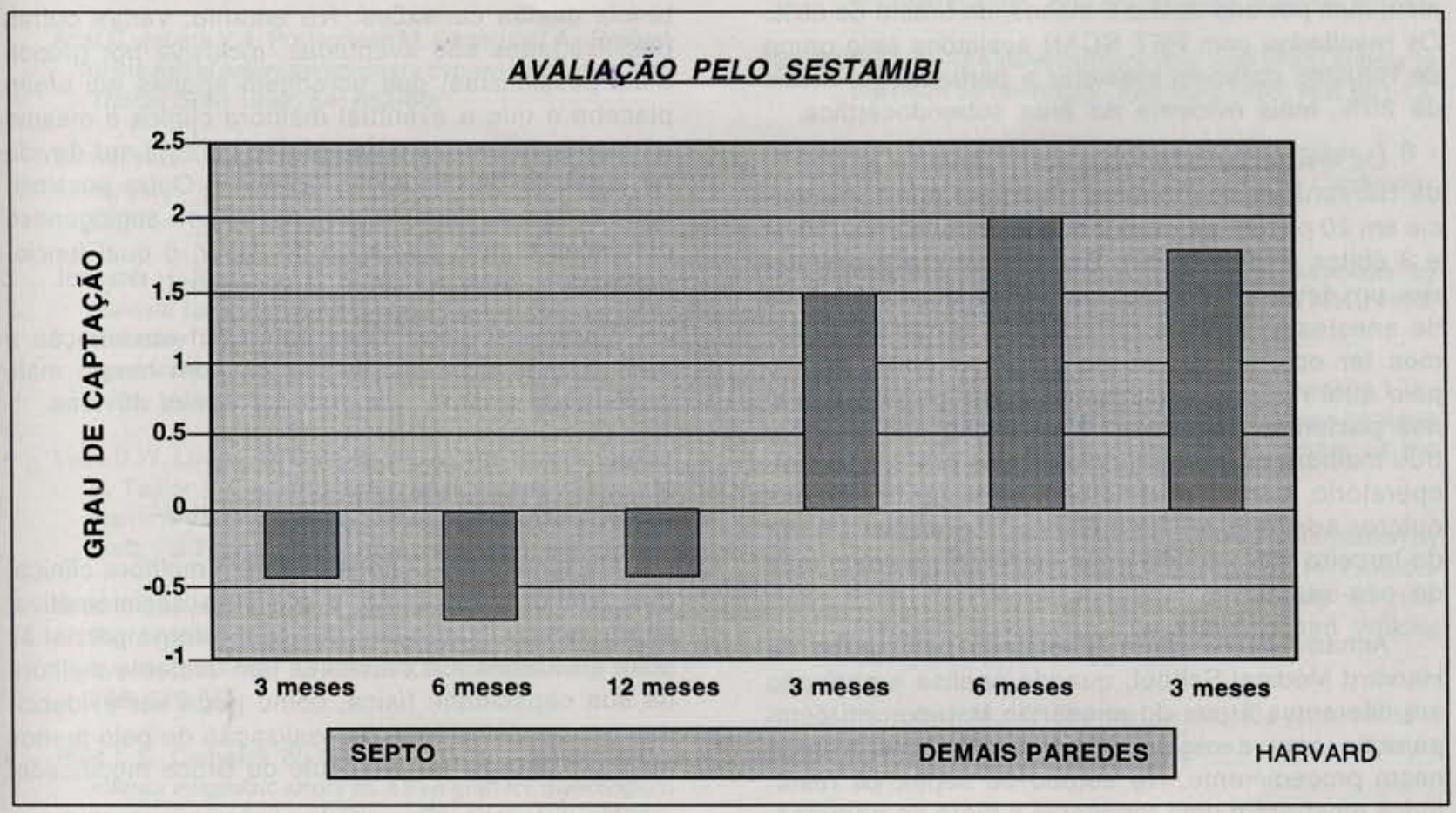

doses de nitrato sublingual e uso de medicação endovenosa, passaram todo o período de pós-operatório sem ocorrência de angina significativa ou uso de nitrato sublingual.

\section{COMENTÁRIOS}

As tentativas para levar sangue diretamente da cavidade cardíaca ao miocárdio, baseadas no aspecto anatômico dos répteis, são citadas em vários trabalhos $(16,23-25)$. Porém os resultados foram insatisfatórios, uma vez que a grande maioria dos túneis não era detectada após certo período de observação. WHITE \& HERSHEY (26) descreveram 1 caso em que 1 paciente triarterial durante a toracotomia apresentou fibrilação ventricular, não revertendo a inúmeros choques. Os autores efetuaram várias perfurações no miocárdio e, após algum tempo, houve a recuperação dos batimentos, seguida da possibilidade de revascularização com sucesso.

MIRHOSEINI et al. (27) no início dos anos 80 , foram os pioneiros nos estudos experimentais com a utilização de disparos de laser de $\mathrm{CO}_{2}$ de alta potência, com bons resultados; suas pesquisas permitiram o uso em pacientes clínicos, de forma combinada à revascularização do miocárdio. Os resultados satisfatórios dessa técnica encontraram restrições quanto à sua eficácia, uma vez que a melhora clínica poderia ser devida ao desenvolvimento de colaterais a partir das pontes tradicionais realizadas.

Outros trabalhos experimentais ressaltam a eficiência do método, o que tem permitido a sua utilização em pacientes de vários grupos, como forma isolada ou associada. Nos Estados Unidos, oito Centros têm realizado esse tipo de cirurgia com protocolos específicos, principalmente no que se refere ao estudo pós-operatório da perfusão miocárdica.

Fora dos E.U.A., outros 17 Centros em vários países têm utilizado o método e um registro internacional, o TMR Registry Report* apresentou, em setembro de 1995 , os resultados de 324 pacientes, sendo $83 \%$ do sexo masculino, com média de idades de 60 anos, sendo $82 \%$ em grupo funcional III ou IV, e $36 \%$ com angina instável. A maioria dos pacientes já havia sido submetida à cirurgia de revascularização do miocárdio. O número de túneis foi em média de 26 a 27, e em geral se utilizou entre 35 e 45 joules. Houve $10 \%$ de óbitos neste grupo de pacientes, sendo a maioria por disfunção de ventrículo esquerdo. Os dados de evolução pósoperatória ainda não foram compilados.

Os resultados clínicos têm sido obtidos através de informaçöes pessoais de grupos como os de Houston, Chicago e do próprio Dr. Mirhoseini, mos-

* TMR Registry Report - Paris - setembro, 1995. 
trando mortalidade em torno de $10 \%$ e melhora clinica, num período de 3 a 6 meses, da ordem de $60 \%$. Os resultados com PET SCAN avaliados pelo grupo de Houston parecem melhorar a perfusão em niveis de $20 \%$, mais evidente na área subendocárdica.

Os resultados publicados por HORVATH et al. (28), da Harvard Medical School, mostram sua experiência em 20 pacientes, com 2 óbitos hospitalares (10\%) e 3 óbitos tardios (15\%). Entre os óbitos hospitalares, um deles foi atribuído ao uso de dose excessiva de anestésico através da epidural, o que suspeitamos ter ocorrido no nosso segundo óbito, motivo pelo qual não mais utilizamos a analgesia epidural nos pacientes seguintes. A evolução clínica mostrou melhora do grupo funcional que era 3,7 no préoperatório, para 1 no pós-operatório tardio. Alguns autores admitem que há melhora progressiva a partir do terceiro mês, sendo mais evidente no sexto mês de pós-operatório.

Achado interessante é relatado pelo grupo da Harvard Medical School, quando analisa a perfusão em diferentes áreas do miocárdio tratado, em comparação com a regiāo septal, que não é tratada neste procedimento. No estudo do septo, os resultados mostraram uma tendência a piora da perfusão, enquanto que as demais paredes denotaram tendência a melhora (Gráfico 3).

Quanto ao mecanismo pelo qual os túneis promoveriam a melhora da perfusão miocárdica, há ainda muita controvérsia. A teoria mais aceita é aquela pela qual, a partir das perfuraçōes, haveria conexão com os sinusóides e destes com as terminações coronarianas. Tal fato é, inclusive, relatado por COOLEY et al. (29), do Texas Heart Institute, que mostram, em estudo anatomopatológico, a existência destas conexões. No entanto, várias outras possibilidades são aventadas, inclusive por grupos mais pessimistas, que acreditam apenas em efeito placebo e que a eventual melhora clínica e mesmo a observada em teste de esforço poderia ser devida ao aumento de circulação colateral. Outra possibilidade seria a do desenvolvimento de neo-angiogenese determinada pela aplicação do laser, o qual funcionaria como uma citocininà angiogênica (30).

Vários aspectos ainda estão em observação e apenas a análise dos pacientes num tempo mais prolongado poderá responder a essas dúvidas.

\section{CONCLUSÕES}

Os resultados obtidos sugerem melhora clínica, que, embora não torne o paciente assintomático, altera sua qualidade de vida, com retorno parcial às suas atividades. Os pacientes têm evidente melhora na sua capacidade física, como pode ser evidenciado pela possibilidade da realização de pelo menos mais um estágio no protocolo de Bruce modificado.

No entanto, os resultados com os testes laboratoriais, ainda em pequeno número de pacientes estudados de forma seqüencial, não permitem uma significância evidente na eventual melhoria da perfusão. Os dados da literatura já começam a denotar efeitos benéficos.

Portanto, há necessidade de maior número de pacientes e de seguimento mais longo, para que possamos ter um julgamento adequado da eficiência do método.

RBCCV 44205-292

Galantier M, Moreira G B, Bub R F, Galantier J, Buffolo E, Carvalho A C, Armaganijan D, Féher J Transmyocardial laser revascularization. Rev Bras Cir Cardiovasc 1996; 11 (2):67-74.

ABSTRACT: Patients with ischemic heart disease and disabling angina, for whom CABG or PTCA is impossible, present a difficult chalenge. The Transmyocardial Laser Revascularization (TMLR) provides direct perfusion of ischemic myocardium vialaser by creating transmural channels. Since April 1995 we have treated 11 patients, 9 males, mean age 68 years, with TMLR. Preoperatively 9 were in angina class IV, despite adequate and even maximum clinical medical treatment. The patients were screened preoperatively by SESTAMIBI perfusion scan and pharmacological echocardiogram to identify the location and extention of their reversible ischemia. Operative exposure was obtained via a left anterior thoracotomy. Employing a $850 \mathrm{~W} \mathrm{CO} 2$ laser an average of 30 was created. Bleeding from the channels was controlled by direct finger pressure and rarely by epicardial suture. The early mortality was 2 out of 11 patients. There was no late mortality. All patients revealed improvement in their clinical status, and the mean angina class was 1,8 postoperatively; In 4 patients SESTAMIBI scan was performed at the third postoperative month, and 1 showed improvement in the myocardium perfusion. Those early results indicate that TMLR is a single operative technique that may improve myocardial perfusion and provide angina relief for patients not amenable to standard methods of revascularization. However a larger number of patients and a longer time of follow-up will be needed to have definitive conclusions.

DESCRIPTORS: Myocardial revascularization, methods. Laser surgery, methods. Myocardial ischemia surgery. Angina pectoris, surgery. Coronary arteriosclerosis, surgery. Coronary atherosclerosis, surgery. 
Galantier M, Moreira G B, Bub RF, Galantier J, Buffolo E, Carvalho A C, Armaganijan D, Féher J-Revascularizaçāo transmiocárdica a laser. Rev Bras Cir Cardiovasc 1996; 11 (2): 67-74.

\section{REFERÊNCIAS BIBLIOGRÁFICAS}

1 Acar C, Jebara V A, Portoghese M, Carpentier A - Revival of the radial artery for coronary bypass grafting. Ann Thorac Surg 1992; 54: 652-60.

2 Barner H B - New arterial conduits for coronary bypass surgery Semin Thorac Cardiovasc Surg 1994; 6: 76-80.

3 Jones R H, Philips H R, Mark D B, et al. - Long-term survival benefit of CABG and PTCA in patients with coronary artery disease. Annual Meeting of the American Association for Thoracic Surgery, 75th 1995. [Abstracts].

4 Lytle B W, Loop F D, Cosgrove D M, Ratliff N B, Easley $\mathrm{K}$, Taylor P C - Long-term serial studies of internal mammary artery and saphenous vein coronary bypass graft. J Thorac Cardiovasc Surg 1985; 89: 615-23.

5 Mills N L, Hockmuth D R, Everson C T, Robart C C Right gastroepiploic artery used for coronary artery bypass grafting. J Thorac Cardiovasc Surg 1993; 106: $579-86$.

6 Puig L B, Ciongolli W, Cividanes G V L, Jatene A D Inferior epigastric artery as a free graft for myocardium revascularization. J Thorac Cardiovasc Surg 1990; 99: 251-5.

7 Suma H, Wanibuchi $Y$, Terada $Y$, Fukuda S, Takayama $T$, Furutas - The right astroepiploic artery graft. $J$ Thorac Cardiovasc Surg 1993; 105: 615-23.

8 Wilson J M \& Ferguson, J J - Revascularization therapy for coronary artery disease. Texas Heart Inst J 1995; 22: $145-61$.

9 Arie S, Garcia P, Brito F S, Caixeta A M, Soares P R, Horta P E - Angioplastia coronária com uso do Laser. Rev Soc Cardiol Estado de Sāo Paulo 1995; 5: 552-9.

10 Mattos L A, Carneiro J K, Feres F, Pinto I, Sousa A G M R, Sousa J E M R - Aterectomia rotacional de alta velocidade. Rev Soc Cardiol Estado de São Paulo 1995; 5: 542-51.

11 Ribeiro E E, Silva L A, Petrizzo A, Barbosa R - O Futuro da aterectomia coronária direcionada. Rev Soc Cardiol Estado de São Paulo 1995; 5: 537-41.

12 Souza A G M R, Pinto I, Tanajura L F et al. - O Stent de Palmaz - Schatz para o tratamento da doença aterosclerótica obstrutiva coronária. Rev Soc Cardiol Estado de São Paulo 1995; 5: 560-5.

13 Christakis G T, Rao V, Fremes S E, Chen E, Naylor C D, Goldman B S - Does coronary endarterectomy adversely affect the results of bypass surgery? J Card Surg 1993; 8: 72-8.

B J - Controversial aspects of coronary endarterectomy. Ann Thorac Surg 1989; 48: 235-41.

15

16

Mirhoseini $M$, Mickerheide $M$, Cayton $M M$ Transventricular revascularization by Laser. Lasers Surg Med 1982; 2: 187-98.

21 Cooley D A, Frazier O H, Kadipasaoglu K A - Myocardial function and infarct size following acute ischemia and TMLR: short and long-term assessment in a canine model. Second Annual Meeting on Transmyocardial Revascularization, 1993. [Abstracts]

Horvath K A, Smith W J, Laurence R G, Schoen F J, Appleyard R F, Cohn L H - Recovery and viability of acute myocardial infarct after transmyocardial Laser revascularization. J Am Coll Cardiol 1995; 25: $258-63$

23 Khazei A H, Kime W P, Papadopoulos C, Cowley R A - Myocardial canalization. Ann Thorac Surg 1968; 6: 163-7.

Kuzela L \& Miller G E - Experimental evaluation of direct transventricular revascularization. J Thorac Cardiovasc Surg 1969; 57: 770-3.

Pifarré R, Jasuja M L, Lynch R D, Neville W E Myocardial revascularization by transmyocardial acupuncture. J Thorac Cardiovasc Surg 1969; 58: 424-31.

White, M \& Hershey J E - Multiple transmyocardial puncture revascularization in refractory ventricular fibrillation due to myocardial ischemia. Ann Thorac Surg 1968; 6: 557-65.

27 Mirhoseini M, Shelgikar S, Cayton M M - New concepts in revascularization of the myocardium. Ann Thorac Surg $1988 ; 45: 415-20$

28 Horvath K A, Mannting F, Cummings N, Shernan S K, Cohn L H - Transmyocardial Laser revascularization: 
operative techniques and clinical results at two years. Western Thorac Surg Assoc Meeting 1995. [Abstracts]

Cooley D A, Frazier O H, Kadipasaoglu K A, Pehjlvanoglu S, Shannon R L, Angelini P. Transmyo-

\section{Discussão}

\section{DR. PAULO ROBERTO BROFMAN Curitiba, $P R$}

Cumprimento o Dr. Maurício e os demais autores, pelo excelente trabalho, correta apresentação e pelo pioneirismo da técnica. Faço questão de estender e, por favor, transmita ao Dr. Jozef Féher um especial cumprimento, pela sua capacidade "visionária", de percepção, trazendo ao País de forma pioneira o que há de mais avançado em tecnologia de ponta no diagnóstico e terapêutica médica. A utilização de raios laser na área médica não é recente e em especial na cardiologia intervencionista. A desobstrução intracoronariana através de cateter com diferentes tipos de raio laser já foi largamente empregada e hoje tem sua indicação bastante limitada pela alta incidência de complicaçōes e insucessos. A utilização de raio lazer de $\mathrm{CO}^{2}$ de alta potência para a revascularização transmiocárdica, porém, baseia-se em principios diferentes e é uma idéia mais promissora que a citada anteriormente. A estatística apresentada pelo Dr. Maurício assemelha-se às apresentadas pelos dez grupos envolvidos na fase inicial de desenvolvimento clínico do método. Em tôdas as conclusões daqueles estudos coincide com a sua, ou seja, o pequeno número de pacientes requer uma melhor observação para quantificar a eficácia do método. Faço duas perguntas, uma vez que, evidentemente, não tenho experiência com a técnica, pois a sua Instituição é a única dotada de tal equipamento no País. $1^{\circ}$ ) Esta técnica não fará reviver a cirurgia de Vineberg, isolada ou associada, uma vez que os critérios de seleção dos pacientes são semelhantes? $2^{\circ}$ ) De cardial Laser revascularization. Texas Heart Inst $J$ 1994; 21: 220-4.

Höckel M, Schlenger K, Doctrow S, Kissel T, Vaupel P - Therapeutic angiogenesis. Arch Surg 1993; 128: 423-9.

acordo com dados da A H A, no ano de 1995 deveriam ser realizados nos Estados Unidos cerca de 1.125 .000 procedimentos de cardiologia intervencionista, assim distribuídos: 570.000 angioplastias, 550.000 revascularizações do miocárdio e 5.000 revascularizações transmiocárdicas e, pelos dados de literatura, não foram realizados mais de 800 casos com esta técnica. A que você atribui estes números? Mais uma vez, meus cumprimentos. Obrigado.

\section{DR. GALANTIER \\ (Encerrando)}

Gostariamos, inicialmente, de agradecer os comentários e, principalmente, a menção ao Dr. Feher, que realmente foi a pessoa que, tomando conhecimento deste equipamento, vislumbrou a possibilidade de sua aquisição pelo Hospital Israelita Albert Einstein, não poupando esforços para a sua concretização. Graças ao seu dinamismo e persistência, estamos tendo a oportunidade do desenvolvimento dessa esperiência clínica. Em relação à sua primeira questão, Dr. Brofman, realmente acreditamos que, para casos que se enquadrem no protocolo de laser, haveria possibilidade também da utilização da cirurgia de Vineberg, e que Serviços que não disponham deste equipamento poderiam usá-la como método alternativo. Inclusive, um estudo interessante seria a comparação entre esses dois métodos. Quanto ao número inferior de procedimentos realizados com laser, acreditamos que se deva ao fato de não existirem equipamentos em número suficiente, além, obviamente, de uma difusão limitada do método e também em função dos resultados ainda não serem totalmente comprovados. 